\title{
Photoelectric Effect at Ultrahigh Intensities
}

\author{
A. A. Sorokin, ${ }^{1,2}$ S. V. Bobashev, ${ }^{2}$ T. Feigl,${ }^{3}$ K. Tiedtke, ${ }^{4}$ H. Wabnitz, ${ }^{4}$ and M. Richter ${ }^{1}$ \\ ${ }^{1}$ Physikalisch-Technische Bundesanstalt (PTB), Abbestraße 2-12, D-10587 Berlin, Germany \\ ${ }^{2}$ Ioffe Physico-Technical Institute, Polytekhnicheskaya 26, 194021 St. Petersburg, Russia \\ ${ }^{3}$ Fraunhofer Inst. f. Ang. Optik u. Feinm., Albert-Einstein-Straße 7, D-07745 Jena, Germany \\ ${ }^{4}$ Deutsches Elektronen-Synchrotron (DESY), Notkestraße 85, D-22603 Hamburg, Germany
}

(Received 20 April 2007; published 20 November 2007)

\begin{abstract}
In the spectral range of the extreme ultraviolet at a wavelength of $13.3 \mathrm{~nm}$, we have studied the photoionization of xenon at ultrahigh intensities. For our ion mass-to-charge spectroscopy experiments, irradiance levels from $10^{12}$ to $10^{16} \mathrm{~W} \mathrm{~cm}^{-2}$ were achieved at the new free-electron laser in Hamburg FLASH by strong beam focusing with the aid of a spherical multilayer mirror. Ion charges up to $\mathrm{Xe}^{21+}$ were observed and investigated as a function of irradiance. Our surprising results are discussed in terms of a perturbative and nonperturbative description.
\end{abstract}

PACS numbers: $32.80 . \mathrm{Rm}, 32.80 . \mathrm{Fb}, 41.60 . \mathrm{Cr}, 42.50 . \mathrm{Hz}$

The photoelectric effect, i.e., the electron emission from matter when ionized by short-wavelength radiation, has been well known for more than 100 years and is widely used as a probe in materials research. The effect is also of fundamental significance because at low intensities, it represents a proof for the quantum structure of light and photons as the light particles [1]. At long-wavelengths, on the other hand, photoelectron emission and photoionization are restricted to ultrahigh photon intensities using, e.g., powerful optical lasers. This process may be described by treating intense light as an electromagnetic wave of high field strength which strongly influences the atomic electron structure [[2,3], and references therein]. The combination of short wavelengths in conjunction with ultrahigh intensities is, however, largely unexplored territory, where the physics of the classical photoeffect meets the regime of strong-field phenomena. Here, as we show, the mechanisms of photon-matter interaction seem not to be completely understood. At the hitherto highest irradiance levels of almost $10^{16} \mathrm{~W} \mathrm{~cm}^{-2}$ in the Extreme Ultra-Violet (EUV) at a wavelength of $13.3 \mathrm{~nm}$, we have observed, by ion mass-to-charge spectroscopy, surprisingly high degrees of photoionization on xenon atoms. Neither a pure particle nor a pure wave picture of light seems to give a satisfying explanation for our experimental results which nicely demonstrates the dual nature of light. The work was performed at the new Free-electron LASer in Hamburg FLASH [4,5] and might be of significance for any investigation at current and future x-ray laser facilities [6-9] on fields like plasma physics, new materials, femtochemistry, and biochemical structure and dynamics.

The almost parallel Free-Electron Laser (FEL) beam of FLASH was used at $60 \mathrm{~m}$ distance from the source point, as schematically shown in Fig. 1. The particular EUV wavelength of $13.3 \mathrm{~nm}$, i.e., the photon energy of $93 \mathrm{eV}$, was chosen because it allowed strong beam focusing by use of a spherical Si/Mo narrow-band multilayer mirror under normal incidence with a focal length of $200 \mathrm{~mm}$ and a reflec- tance of $68 \%$ as has been developed for industrial applications within the framework of EUV lithography [10]. The full-width at half maximum focus diameter of $(2.6 \pm 0.5) \mu \mathrm{m}$ was derived from the target depletion effect [11]. FEL pulse energy $W$ in the microjoule regime was monitored on a shot-to-shot basis with a relative uncertainty of $15 \%$ by means of a gas-monitor detector [12]. The estimated FEL pulse duration amounted to $\tau=$ $(10 \pm 2)$ fs [5]. The EUV mirror could be moved by $\pm 2 \mathrm{~cm}$ along the photon beam in order to vary the beam cross section $A$ within the interaction zone and, by that, the pulse irradiance $E=W /(A \times \tau)$ in the range from $10^{12}$ to $10^{16} \mathrm{~W} \mathrm{~cm}{ }^{-2}$. Pulse-to-pulse intensity fluctuations varied from 35 to $45 \%$. To ensure that any interaction between neighboring atoms do not affect our data, the target gas filled the experimental vacuum chamber homogeneously at the considerably low pressure of about $1.1 \times 10^{-4} \mathrm{~Pa}$. Some of the measurements were repeated at $0.6,1.7$, and $2.0 \times 10^{-4} \mathrm{~Pa}$ without any significant change of the data. The target gas pressure was measured and controlled by a calibrated spinning rotor vacuum gauge, the temperature by a calibrated Pt100 resistance thermometer. In front of the chamber, a horizontal beam stop of $1.5 \mathrm{~mm}$ in height

\section{ion TOF spectrometer}

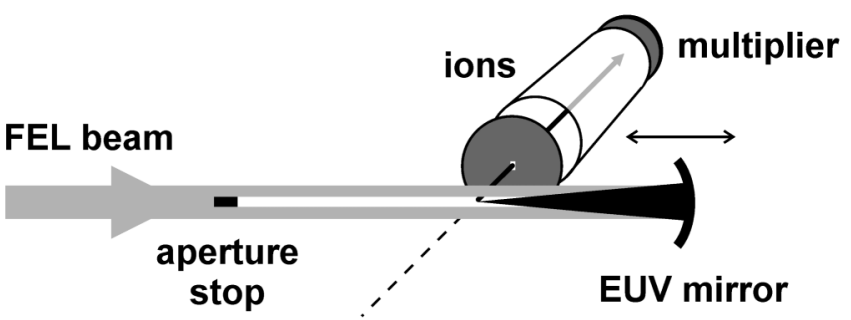

FIG. 1. Experimental setup for the investigation of atoms by ion time-of-flight (TOF) spectroscopy in a Free-Electron Laser (FEL) focus of a spherical Extreme Ultra-Violet (EUV) multilayer mirror. 
was introduced. The entrance aperture of our ion time-offlight (TOF) mass-to-charge spectrometer had, on the other hand, an extension of $350 \mu \mathrm{m}$ in beam direction and $1.0 \mathrm{~mm}$ perpendicular to the photon beam. Hence, due to a very homogeneous extraction field, ions produced by the incident unfocused radiation were diaphragmed as shown in Fig. 1. Behind the drift section of the TOF spectrometer, absolute ion detection was realized by an open multiplier $[11,13,14]$. The pulse-resolved ion-TOF measurements were performed with respect to the FEL bunch clock pulses by means of a digital oscilloscope. TOF spectra were averaged over typically 300 to 500 consecutive photon shots. Xenon (Xe) gas was considered to be the most suited target to study the atomic effects on strong photon-matter interaction in the EUV because of its so-called $4 d$ giant resonance in this photon energy range [15].

Figure 2 shows ion TOF spectra of Xe atoms taken at different pulse irradiance levels covering the range from one-photon excitation to the strong-field regime. The data are not affected by plasma or other next neighbor effects such as electron impact ionization because we did not observe any line broadening or dependence on the target gas pressure. In the spectral range of optical radiation, strong-field phenomena are commonly described by socalled nonperturbative theories within the wave picture of light. Their applicability depends, however, on the ponderomotive energy $U_{p}$ which is the quiver energy transferred to an electron by the oscillating field of an electromagnetic wave $[2,3] . U_{p}$ increases linearly with the irradiance $E$, but decreases with the square of the photon energy $\hbar \omega$ :

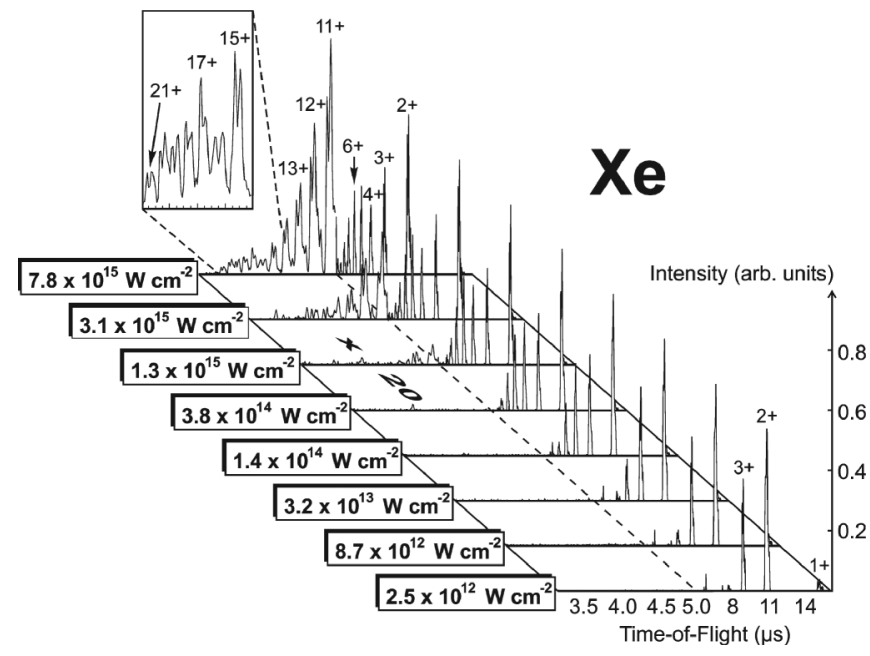

FIG. 2. Ion time-of-flight (TOF) mass/charge spectra of xenon (Xe) taken at a photon energy of $93 \mathrm{eV}$ and different pulse irradiance levels. Each spectrum represents an accumulation over 300 to 500 consecutive FEL shots. In the low TOF regime (below $5 \mu \mathrm{s}$ ), the ion intensities were multiplied by a factor of 20. The multiplet structures of the different ion signals are due to the Xe isotope distribution.

$$
U_{p} / \mathrm{eV} \approx 1.44 \frac{E /\left(10^{13} \mathrm{~W} \mathrm{~cm}^{-2}\right)}{(\hbar \omega / \mathrm{eV})^{2}}
$$

At the high photon energy of $93 \mathrm{eV}, U_{p}$ does not exceed a value of $0.2 \mathrm{eV}$ for the present experiments, not even at our highest irradiance level of $7.8 \times 10^{15} \mathrm{~W} \mathrm{~cm}^{-2}$. Thus, $U_{p}$ is by orders of magnitude lower than the photon energy and the Xe ionization energy of $12.1 \mathrm{eV}$, which might indicate that a nonperturbative approach as applied for strong-field phenomena in the optical regime is not appropriate for the present results.

To discuss the series of ion spectra in Fig. 2 within the framework of perturbation theory and photons as the light particles, we start at low irradiance. The main process of one-photon excitation of $\mathrm{Xe}$ (ground state electron configuration $\mathrm{Xe}:[\mathrm{Kr}] 4 d^{10} 5 s^{2} 5 p^{6}$ ) at $93 \mathrm{eV}$ is resonant photoelectron emission from the inner $4 d$ electron shell. This process is well known as the giant $4 d \rightarrow \varepsilon f$ continuum resonance [15] and leads via Auger decay to doubly or triply charged final states with two or three electron vacancies in the outer $5 p$ shell, respectively [16]. Direct $5 p$ single ionization plays a minor role. These processes are depicted on the left side of Fig. 3 and are confirmed by our low-irradiance ion spectrum at the bottom of Fig. 2. However, with increasing irradiance, additional higher charges occur which can only be explained by higher-order multiphoton effects.

As has recently been demonstrated at FLASH, the multiphoton ionization at photon energies well above atomic (and ionic) ionization thresholds and at photon pulse intensities of soft-x-ray FELs is strongly affected by sequences of one-photon processes in which an ion created in a preceding step represents a new target for a subsequent step $[13,14]$. According to this scheme, one-photon ionization starting from the ground state of $\mathrm{Xe}^{1+}$ created in a first step

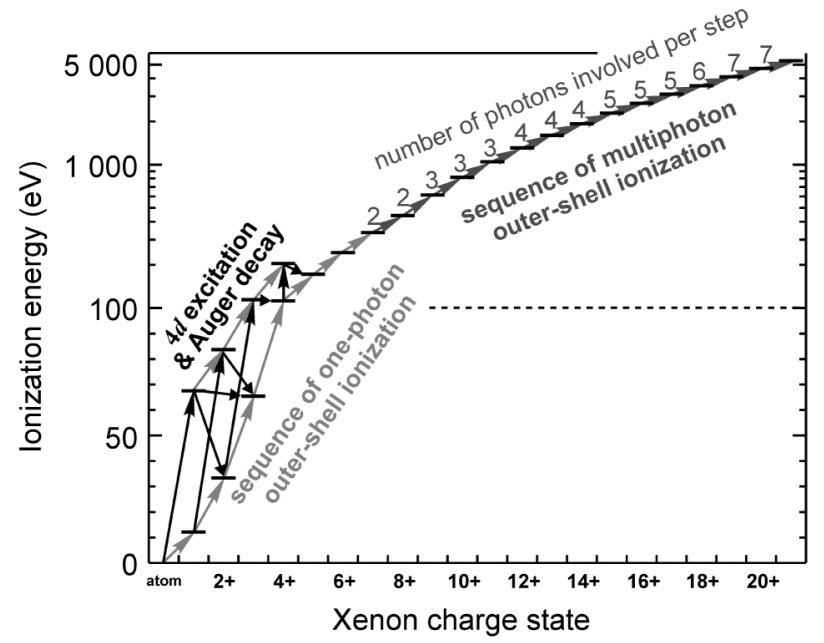

FIG. 3. Energy levels and sequential multiphoton ionization channels via ionic states for xenon at a photon energy of $93 \mathrm{eV}$. 
is also dominated by a giant $4 d \rightarrow \varepsilon f$ excitation [17]. Subsequent Auger decay results in $\mathrm{Xe}^{3+}$ (see Fig. 3) $[18,19]$. The same holds for $\mathrm{Xe}^{2+}$, leading to $\mathrm{Xe}^{4+}[18]$. As a competing process, one-photon ionization in the outer $5 p$ shell may have certain relevance, starting from the ionic ground state configuration and/or from a $4 d$ excited state, respectively. The $4 d$ ionization threshold of $\mathrm{Xe}^{3+}$ is beyond the EUV photon energy of $93 \mathrm{eV}[20,21]$ so that direct $5 p$ photoionization should be even the most probable channel from $\mathrm{Xe}^{3+}$ to $\mathrm{Xe}^{4+}$. In this manner, the complete outer $5 p^{6}$ shell can be removed, thus enabling $\mathrm{Xe}^{6+}$ to be reached. However, $5 p$ photoionization of $\mathrm{Xe}^{4+}$ might be resonantly enhanced by a discrete autoionizing $4 d \rightarrow 4 f$ resonance (see Fig. 3) [21,22]. The situation is even more complicated if a first photoionization process in an inner $4 d$ - (or $5 s-$ ) shell is followed by a second or third, in competition with Auger decay, leading to a sort of "hollow" atom. Those processes are not shown in Fig. 3 but might be relevant since Auger decaying excited states are expected to have lifetimes in the order of the FLASH pulse duration of 10 fs $[5,23]$.

In the ground state of $\mathrm{Xe}^{6+}$, the outer shell is represented by the two $5 s$ electrons. While the ionization energy of the first amounts to $92.1 \mathrm{eV}$, i.e., slightly less than our EUV photon energy of $93 \mathrm{eV}$, excitation of the second $5 s$ electron requires $105.9 \mathrm{eV}$ for the corresponding $\mathrm{Xe}^{7+} \rightarrow$ $\mathrm{Xe}^{8+}$ process [24], i.e., at least two EUV photons. Hence, the latter transition has a new quality. Continuing, for the $\mathrm{Xe}^{8+} \rightarrow \mathrm{Xe}^{9+}$ transition, again two EUV photons are required. From $\mathrm{Xe}^{10+}$ to $\mathrm{Xe}^{12+}$, the number of EUV photons involved to create the respective ion from the preceding charge state must even be at least three. From $\mathrm{Xe}^{13+}$ to $\mathrm{Xe}^{15+}$, this number amounts to four, from $\mathrm{Xe}^{16+}$ to $\mathrm{Xe}^{18+}$ to five, for $\mathrm{Xe}^{19+}$ to six, and for $\mathrm{Xe}^{20+}$ and $\mathrm{Xe}^{21+}$, finally, to seven, as shown in Fig. 3. The respective multiphoton ionization processes might be further influenced by discrete $4 d \rightarrow 5 p$ inner-shell excitations which are expected for higher charge states to occur around $93 \mathrm{eV}$ photon energy. In this manner, obviously, a rough explanation for the series of ion spectra shown in Fig. 2 is given within the particle picture of the radiation field which is in accordance with former works on rare gases at lower photon energies and irradiance levels compared to the present work [14,25-30].

However, in $\mathrm{Xe}^{21+}$ as observed in the ion spectrum of the inset of Fig. 2 at an EUV irradiance of almost $10^{16} \mathrm{~W} \mathrm{~cm}^{-2}$, the complete $5 p^{6}, 5 s^{2}$, and $4 d^{10}$ electron shells have disappeared in the ground state and three $4 p$ electrons as well. A corresponding perturbative approach has to consider about 19 steps to generate $\mathrm{Xe}^{21+}$ from atomic Xe (see Fig. 3) which is related to 19 coupled differential rate equations with 19 individual multiphoton ionization cross sections. As yet, such a theory, which in an appropriate manner takes electron-electron correlation into account, does not exist and seems to be a challenging task.
Moreover, for the generation of $\mathrm{Xe}^{21+}$, a total energy of more than $5 \mathrm{keV}$ must have been absorbed by a single atom within a photon pulse duration of $10 \mathrm{fs}$, i.e., more than 57 EUV photons of $93 \mathrm{eV}$ photon energy. Hence, the situation might even be beyond the scope of the perturbation theory and the particle picture of light.

In this context, it is interesting to compare our data with the results which have recently been obtained from the multiple ionization of Xe by superstrong optical radiation as emitted from a titan-sapphire femtosecond laser at the wavelength of $800 \mathrm{~nm}$ and, hence, the low photon energy of $1.55 \mathrm{eV}$ [24]. Here, according to Eq. (1), the ponderomotive energy is generally higher by a factor of $(93 \mathrm{eV} / 1.55 \mathrm{eV})^{2} \approx 3500$ than in the EUV, which suggests nonperturbative description at irradiance levels around $10^{16} \mathrm{~W} \mathrm{~cm}^{-2}$. The degree of photon-matter interaction, on the other hand, seems not to be higher because at $10^{16} \mathrm{~W} \mathrm{~cm}^{-2}$ of optical radiation, an ionization only up to $\mathrm{Xe}^{8+}$ is obtained, whereas in the EUV, we have observed $\mathrm{Xe}^{21+}$ (Fig. 2). For ionic states up to $\mathrm{Xe}^{15+}$, we were even able to essentially disentangle the signals from residual gas lines and the weaker isotope lines of the respective neighbors in the ion-TOF spectra. This allowed us, in order to gain further insight into the ionization process, to quantitatively evaluate the corresponding ion signal intensities.

In Fig. 4, the ion signal intensities $N_{q_{+}}(1 \leq q \leq 15)$ are displayed as a function of the peak irradiance resulting from averaging over consecutive FLASH pulses. The data are normalized to the number $N$ of initial atomic targets within the interaction volume, respectively, and are given on a double logarithmic scale. For our discussion, the respective initial slopes are of particular interest according to $[2,3]$

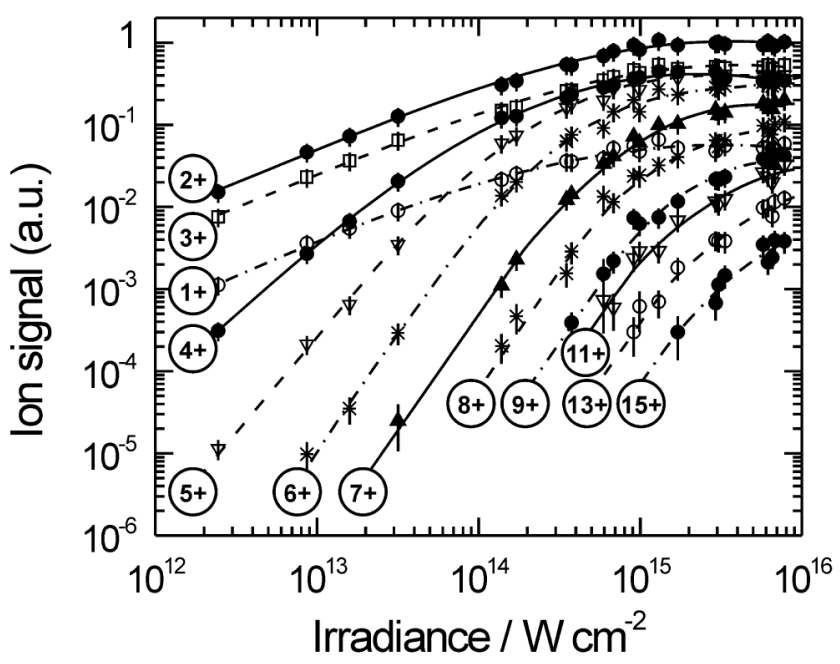

FIG. 4. Relative $\mathrm{Xe}^{q+}$ ion signal intensities as a function of the peak irradiance for different charge states $1 \leq q \leq 15$ at the photon energy of $93 \mathrm{eV}$. The respective fit curves guide the eye through the experimental data points. 


$$
\log \left(\frac{N_{q+}}{N}\right)=C+n \log \left(\frac{E}{\mathrm{~W} \mathrm{~cm}^{-2}}\right)
$$

with $C$ being a constant and $n$ the mean number of photons involved per absorption process. In detail, the initial slope in the order of 1 for $N_{1+}, N_{2+}$, and $N_{3+}$, respectively, demonstrates proportionality between the $\mathrm{Xe}^{1+}, \mathrm{Xe}^{2+}$, and $\mathrm{Xe}^{3+}$ generation and confirms their main origin from one-photon processes as discussed above. The saturation of ion signals at high irradiance is due to target depletion $[11,13,14]$. For intermediate charges $(4 \leq q \leq 6)$, then, the initial slope increases systematically, which suggests that more and more photons are involved in the corresponding process. The fact that $n$ increases with the charge of created ions confirms that the respective multiphoton ionization process being explained by a sequence of more and more one-photon excitations, as discussed above (Fig. 3). However, this tendency ends already at $\mathrm{Xe}^{6+}$, i.e., where the outer $5 p^{6}$ shell is completely removed. For higher charge states and inner shells being involved $(7 \leq q \leq 15)$, the initial slope in the double logarithmic presentation is almost constant. This is in clear contradiction to the scheme of a sequence of multiphoton processes within the framework of perturbation theory and the particle picture of light as depicted in Fig. 3. For the higher charges, our results resemble rather the behavior in the optical strong-field regime where the initial slope of the ion signal dependencies on irradiance does not vary so much for the different charge states, and ion generation is well described by nonperturbative theories within the wave picture of light [24].

From our photoionization experiments, we can, therefore, conclude that the appropriate theoretical ansatz to describe photon-matter interaction in the extreme ultraviolet at ultrahigh photon intensities is not clear. Pure perturbative or nonperturbative approaches do not seem to well explain our experimental findings of extreme ionization of xenon. As such, our experimental work may be the starting point for new theoretical work on photon-matter interaction at short wavelengths and high photon intensities with strong impact on future applications of large x-ray laser facilities.

We thank the FLASH team for the very successful operation of the FEL; we also thank J.R. Schneider,
B. Sonntag, and G. Ulm for continuous support.

[1] A. Einstein, Ann. Phys. (Leipzig) 322, 132 (1905).

[2] M. Protopapas, C. H. Keitel, and P. L. Knight, Rep. Prog. Phys. 60, 389 (1997).

[3] N. Delone and V. Krainov, Multiphoton Processes in Atoms (Springer, New York, 2000).

[4] V. Ayvazyan et al., Eur. Phys. J. D 37, 297 (2006).

[5] W. Ackermann et al., Nat. Photon. 1, 336 (2007).

[6] P. G. O'Shea and H. P. Freund, Science 292, 1853 (2001).

[7] H. C. Kapteyn and T. Ditmire, Nature (London) 420, 467 (2002).

[8] J. Feldhaus, J. Arthur, and J. B. Hastings, J. Phys. B 38, S799 (2005).

[9] T. Sekikawa, A. Kosuge, T. Kanai, and S. Watanabe, Nature (London) 432, 605 (2004).

[10] T. Feigl, S. Yulin, N. Benoit, and N. Kaiser, Microelectron. Eng. 83, 703 (2006).

[11] A. A. Sorokin et al., Appl. Phys. Lett. 89, 221114 (2006).

[12] M. Richter et al., Appl. Phys. Lett. 83, 2970 (2003).

[13] A. A. Sorokin, S. V. Bobashev, K. Tiedtke, and M. Richter, J. Phys. B 39, L299 (2006).

[14] A. A. Sorokin, S. V. Bobashev, K. Tiedtke, M. Wellhöfer, and M. Richter, Phys. Rev. A 75, 051402(R) (2007).

[15] V. Schmidt, Rep. Prog. Phys. 55, 1483 (1992).

[16] T. Luhmann et al., Phys. Rev. A 57, 282 (1998).

[17] M. Sano et al., J. Phys. B 29, 5305 (1996).

[18] P. Andersen et al., J. Phys. B 34, 2009 (2001).

[19] Y. Itoh et al., J. Phys. B 34, 3493 (2001).

[20] E. D. Emmons et al., Phys. Rev. A 71, 042704 (2005).

[21] J. M. Bizau et al., Phys. Rev. A 73, 022718 (2006).

[22] A. Aguilar et al., Phys. Rev. A 73, 032717 (2006).

[23] P. Lablanquie et al., Phys. Rev. Lett. 87, 053001 (2001).

[24] K. Yamakawa et al., Phys. Rev. Lett. 92, 123001 (2004).

[25] Y. Nabekawa, H. Hasegawa, E. J. Takahashi, and K. Midorikawa, Phys. Rev. Lett. 94, 043001 (2005).

[26] H. Wabnitz et al., Phys. Rev. Lett. 94, 023001 (2005).

[27] E. P. Benis, D. Charalambidis, T. N. Kitsopoulos, D. D. Tsakiris, and P. Tzallas, Phys. Rev. A 74, 051402(R) (2006).

[28] M. Nagasono et al., Phys. Rev. A 75, 051406(R) (2007).

[29] R. Moshammer et al., Phys. Rev. Lett. 98, 203001 (2007).

[30] P. Lambropoulos, L. A. A. Nikolopoulos, and M. G. Makris, Phys. Rev. A 72, 013410 (2005). 